\title{
Design of distributed propulsion system for general aviation airplane
}

\author{
Pavel Hospodár ${ }^{1}$, Jan Klesa ${ }^{1}$, and Nikola Žižkovský ${ }^{1, *}$ \\ ${ }^{1}$ CTU Prague, Department of Aerospace Engineering, 16607 Praha 6, Czech Republic
}

\begin{abstract}
In this paper, a small airplane is redesigned by using a distributed electrical propulsion (DEP) system. The design procedure is focused on the reduction of fuel consumption in cruise regime with constrained parameters of take-off/landing. In this case, a one half wing area compared to an original airplane is used. Take-off distance and minimum airspeed for landing is achieved by distributed propellers mounted on the leading edge of the wing. These propellers induce velocity on the wing and thereby increase local dynamic pressure, thus the required lift force can be reached with smaller wing area. Moreover, the distributed propellers are assumed as folded in cruise regime to minimize drag when the main combustion engine provides sufficient power.
\end{abstract}

\section{Introduction}

Distributed electric propulsion (DEP) are often used to design new kind of airplane in the last decade [1],[2]. It enables to create high efficiency airplane in cruise regime and provides extremely short take-off and landing distance [3]. In this paper a different approach is used. In this paper the real general aviation airplane is used to redesign wing to use of DEP. Current fuselage, horizontal and vertical tails and propulsion system are used, only wing is modified.

It is a kind of hybrid power system [4], where distributed propellers are electrically powered and original combustion engine, located in the front of fuselage, provides the required amount of power for cruise regime. The power of DEP system in the takeoff/landing regime is provided from a battery, while those are charged in cruise regime or at ground.

The weight of batteries, engines, electrical controllers and fuel are assumed in the optimization procedure which is using response surface optimization. Design of propeller and their characteristics are computed by Blade element momentum theory and the propeller-wing interaction is calculated by lifting line theory in a non-linear form [5]. It offers a design of multi-propeller wing with high lift devices. Optimal configurations are compared with results of CFD calculations for main regimes.

One of the main advantages of DEP airplane is high aerodynamic efficiency in cruise regime. For that reason, a different smaller wing area geometry was calculated to reach

* Corresponding author: Nikola.Zizkovsky@ffs.cvut.cz 
optimal solution. Unfortunately, smaller wing area directly influences a total lift force and is defined as follows:

$$
\mathrm{L}=\frac{1}{2} \rho v_{0}^{2} c_{L} S_{1}
$$

where $\rho$ is air density, $v_{0}$ is freestreem airpeed, $c_{L}$ is lift coefficient and $S_{1}$ is original wing area. Thanks to DEP we can reach required total lift force for smaller wing area due to velocity $v_{i}$ induced by propellers:

$$
\mathrm{L}=\frac{1}{2} \rho\left(v_{0}+v_{i}\right)^{2} c_{L} S
$$

\section{Cruise wing selection}

As mentioned in the introduction, four different wings are computed to find out the best solution for cruise regime. After that a flight performance is calculated to determine a fuel consumption and finally a DEP system is defined.

\subsection{Wing aerodynamic characteristics}

A low and high-fidelity computational method are used for that purpose. Lifting line theory as low fidelity method is used for calculation of wings aerodynamic characteristic in preliminary design. The geometries are created for constant wing span and taper ratio and four different areas. Geometry of wings are depicted in the following figure:

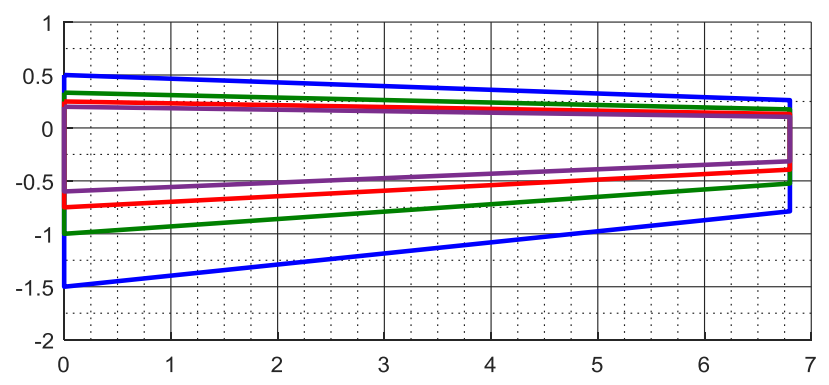

Fig. 1. Geometry of wings with different area

As baseline geometry is used wing of general aviation 10 seater aircraft with weight $2500 \mathrm{~kg}$, cruise speed $400 \mathrm{kph}$ at FL200 and expected range $2500 \mathrm{~km}$. The whole aircraft with zeroed elevators deflection, original and half scaled wing was simulated with CFD as high-fidelity method. Distributed propulsion system with nacelles is applied on half sized wing only. The geometry is intended as symmetrical, so only half of wing is simulated.

As solver is used Open Foam with Reynolds Averaged Simulation (RAS/RANS) model with turbulence modeled by Spalart-Allmaras model applied on compressible steady flow. Boundary layer is solved directly without wall functions. In computational mesh is applied mesh refinement behind wing in length of two chords, which impacts drag characteristic and decay of swirl behind actuator disc. The computational mesh is represented by snapped hex mesh, a hexa-dominant mesh with hanging nodes, combined with prism cells near wall boundary conditions. Number of cells in generated meshes varies from 12 to 17 millions cells, depending on geometry and angle of attack.

Propulsion is simulated with actuator disc method with customized interpretation. In this interpretation radial chord and twist distribution is defined. On half sized wing, ten 
propulsors is applied in constant distance from leading edge. In center of each propulsor, the nacelles are modelled as rounded cylinders with constant diameter, length and local position referenced to local leading edge position.

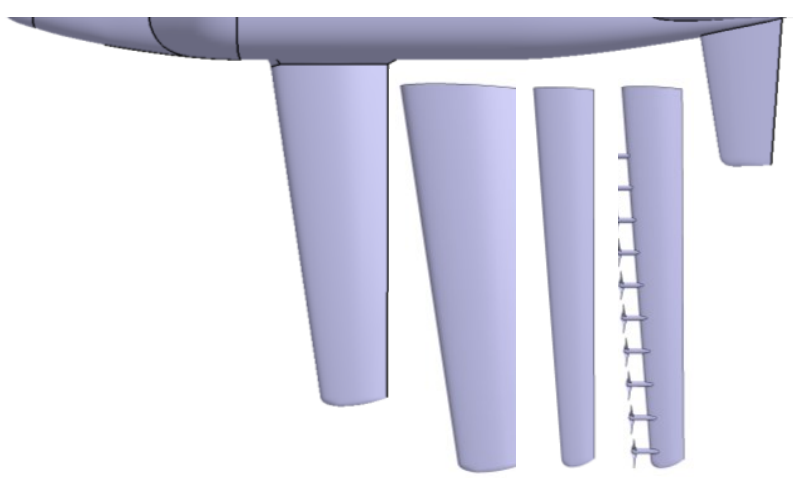

Fig. 2. Geometries used for CFD calculations

With respect to computational demands were calculated just several points of aerodynamic polar. Integral characteristic of whole aircraft with original sized wing were compared to characteristics obtained by measurement in wind tunnel on model of whole aircraft in scale 1:6.5. The differences between wind tunnel experiment and calculated characteristics are given by different slightly different geometry in wing fuselage junction and wing tips, where original wingtips were equipped with winglets. Comparison between results from low and high-fidelity method gives good coincidence.
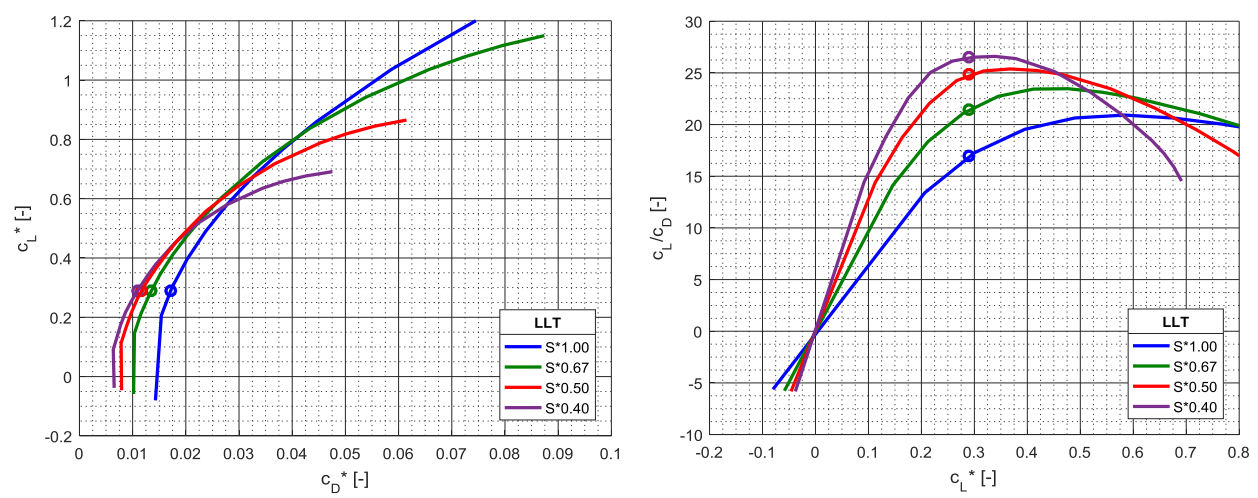

Fig. 3. Aerodynamic characteristics of wings with different calculated by lifting line theory

\subsection{Fuel saving and mass consequences}

To determine a total fuel saving of aircraft with smaller wing an iterative program is developed. In each second a required lift is calculated for specified mass. Then aerodynamic drag is interpolated and required power is determined. Aerodynamic drag of airplane is calculated as a sum of aerodynamic drag of the wing and fuselage. After that a fuel consumption is calculated based on specific fuel consumption of PT6A-42 jet engine which is $0.366 \mathrm{~kg} / \mathrm{kWh}$. New weight of airplane is computed and process are repeated until reaching of range $2500 \mathrm{~km}$. 
This process is used for original aircraft and aircraft with half wing's area and it results to fuel consumption $690 \mathrm{~kg}$ of airplane with original wing and $428 \mathrm{~kg}$ for half wing area. It is around $38 \%$ fuel saving related to original airplane. The saving mass of $262 \mathrm{~kg}$ is divided following manner. $45 \%(118 \mathrm{~kg})$ of saving mass is determined to additional payload and $55 \%(144 \mathrm{~kg})$ is used for purpose of DEP system.

Current DEP system consists of engines, electronic speed controllers (ECS), battery storages and cables. All of these parts can be defined by specific mass - as a ratio to its weight.

EMRAX $(\mathrm{U}=400 \mathrm{~V})[6]$ engine is selected in this example because of its high voltage and has power to weight ratio $\dot{m}_{e}=5.8 \mathrm{~kW} / \mathrm{kg}$. ECS is defined by following power to weight ratio $\dot{m}_{E S C}=30 \mathrm{~kW} / \mathrm{kg}$. The batteries are used only in landing regime and they are assumed full charged. Research of battery predicts a relatively large increasing of energy per kilo, but today best values are around $300 \sim 400 \mathrm{~kW} / \mathrm{kg}$. We choice commonly used battery with $20 \mathrm{C}$ current discharging and its capacity to weight ratio is $\dot{m}_{B}=165 \mathrm{kWh} / \mathrm{kg}$. Last part of DEP system are cables. These are defined as current-weight ratio $\dot{m}_{C}=2000$ $\mathrm{A} /(\mathrm{kg} . \mathrm{m})$. In this point an equation to determine mass distribution of all segments are defined as follows:

$$
m_{0}=\frac{P}{\dot{m}_{e}}+\frac{P}{\dot{m}_{E S C}}+\frac{P \cdot t}{\dot{m}_{B}}+\frac{P}{\dot{m}_{C}} \cdot \frac{d}{U}
$$

where $P$ is total power suitable for DEP, $t$ is time of DEP usage (it is assumed 120 seconds), $d$ is length of electrical cables and $U$ is voltage. In this case a mass used for DEP system is $m_{0}=144 \mathrm{~kg}$. Previous equation has only one unknow - power and can be easily solved. The solution is depicted in following picture.

Total mass: $144.0[\mathrm{~kg}]$

\section{DEP running time: 120 [s]}

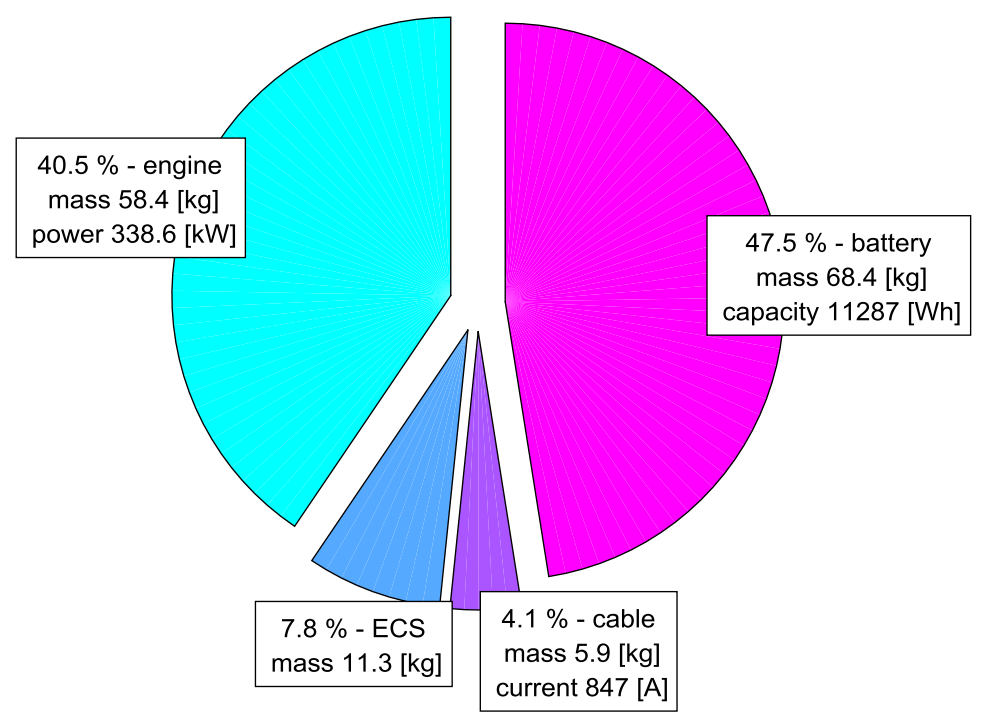

Fig. 4. Mass distribution of DEP system 


\section{Design of distributed system}

In this chapter a design of system that enables to get landing characteristics for smaller wing. Analytical approach to define number of propeller and their size is developed. At the end an aerodynamic characteristic of wing with distributed electric propulsion is computed.

\subsection{DEP sizing}

Design of distributed electric propulsion system is based on landing requirement and suitable power for electric engine. Required value that has to be reached is total lift at landing speed. Equation that describes lift for original airplane and half wing area airplane is described follows:

$$
\frac{1}{2} \rho v_{0}^{2} c_{L} S_{r e f}=\frac{1}{2} \rho\left(v_{0}+v_{i}\right)^{2} c_{L} \frac{S_{r e f}}{2}
$$

where $S_{\text {ref }}$ is area of original wing, $v_{0}$ is freestream velocity and $v_{i}$ is propeller induced velocity. After simplification we get value of required induced velocity:

$$
v_{i}=v_{0}(\sqrt{2}-1)
$$

Using an actuator disk theory, we can derive thrust of propeller:

$$
T=\dot{m}\left(v_{\text {out }}-v_{0}\right)=\rho A v_{\text {disk }}\left(v_{\text {out }}-v_{0}\right)
$$

where $\rho$ is air density, $A$ is actuator disk area, $v_{\text {disk }}$ is velocity in the actuator disk, $v_{\text {out }}$ is velocity behind the propeller and $v_{0}$ is freestream velocity. Further power per disk is determined as:

$$
P_{p}=\eta_{\text {prop }} T v_{\text {disc }}
$$

In the next step a geometry relation between wing span, propeller disk area and number of propellers are defined. Radius of propeller is given by:

$$
r=\frac{l}{2 n}
$$

where $l$ is semi-span wing without fuselage and $n$ is number of propellers per semi-span. Then a propeller area can be defined as:

$$
A=\pi r^{2}=\frac{\pi}{4} \frac{l^{2}}{n^{2}}
$$

Total power for DEP $P_{t o t}$ is $338 \mathrm{~kW}$ (Fig. 3). Half of this power is used for semi-span:

$$
\frac{P_{\text {tot }}}{2}=P_{p} n=\eta_{\text {prop }} T v_{\text {disc }} n
$$

The number of propellers is defined from previous equation after substitution of thrust from eq. 6 and propeller area from eq. 9:

$$
\frac{P_{\text {tot }}}{2}=\eta_{\text {prop }} \rho \frac{\pi}{4} \frac{l^{2}}{n^{2}} v_{\text {disk }}^{2}\left(v_{\text {out }}-v_{0}\right) n
$$


After rearrangement of previous equation and substitution of $v_{\text {out }}=2 v_{1}-v_{0}$ and substitution of $v_{1}=v_{0}+v_{i}$ we get following equation:

$$
n=\eta_{\text {prop }} \frac{\rho \pi l^{2}}{P_{\text {tot }}} v_{i}\left(v_{0}+v_{i}\right)^{2}
$$

Now we substitute equation 5 into previous form and after rearrangement we get final equation for determining of the number of propellers for half area wing:

$$
n=\eta_{\text {prop }} \frac{\rho \pi l^{2}}{P_{\text {tot }}} 2 v_{0}^{3}(\sqrt{2}-1)
$$

\subsection{Propeller design}

Method used for propeller aerodynamic design was described in [9]. It is simple but efficient method. It is based on the combination of the blade element momentum theory (BEMT), Betz's law (detailed description can be found in [7]) and Prandtl's loss function (detailed description in appendix to [7]). It represents simplified version of the Goldstein's method [8]. Input parameters for the computation are engine power, propeller diameter, RPM, number of blades, flight velocity, airfoil characteristics in design point and air density. The outputs are blade twist and chord distribution along the blade. It is depicted the propeller geometry generated by Larrabee method in the following picture as a function of propeller span

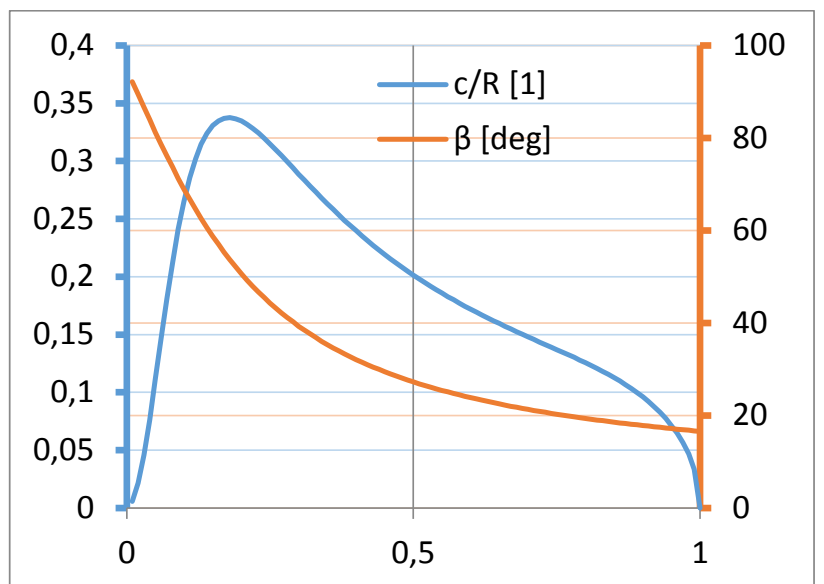

Fig. 5. Propeller geometry designed by Larrabee method

\subsection{Aerodynamic of wing with DEP}

Combination of lifting line theory and Blade element theory is used to estimated propeller wing interaction. This methodology was designed and validated [5]. Development of slipstream propeller velocity is assumed in this method. Classic lifting line theory is adjusted to using an external two dimensional airfoil characteristics. It enables to estimate maximum lift coefficient and total aerodynamic drag (induced, pressure and viscous part). After that, a CFD calculation was done to investigate local characteristics, that you can see in the following figure. 


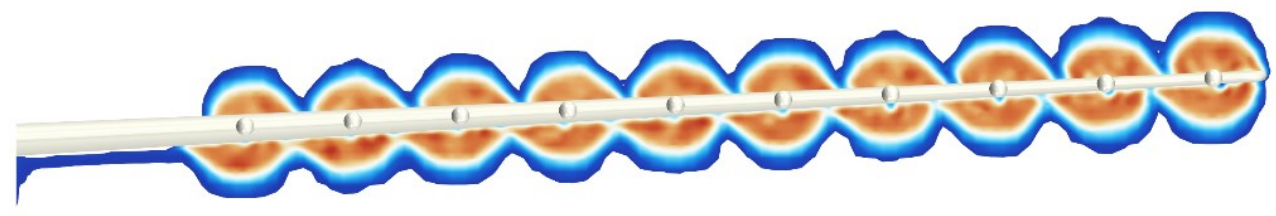

Fig.6. Distributed propulsion on scaled wing geometry - front view - velocity section behind wing

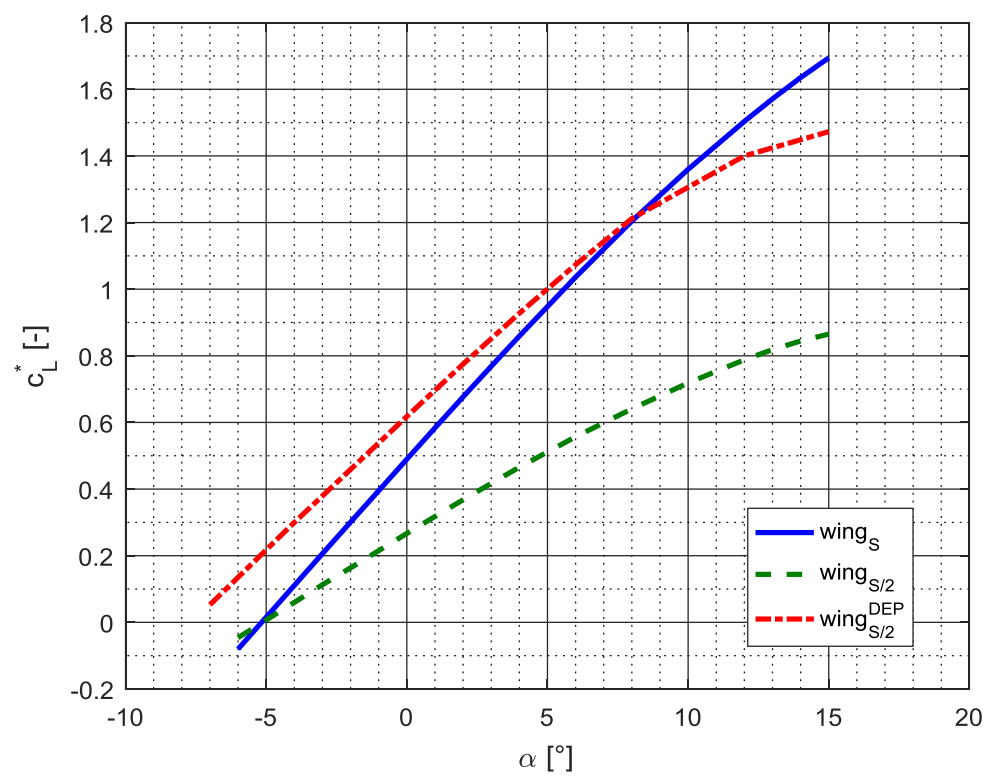

Fig.7. Lift coefficient for different wing geometry a regime

Three different lift coefficients are depicted in previous characteristic. The solid line is lift generated by original wing. Approximately half lift is provided by wing with half area (green line) related to original wing. Finally, half area wing with designed distributed system (red line) provides roughly same lift like original entire wing. This can be considered as proof of concept.

\section{Conclusion}

In this paper the design procedure of distributed electrical propulsion system is presented. Plain airfoil is considered in this work. Design of DEP with high lift devices will be solved in the future.

The integral characteristics of whole aircraft, original wing, half sized wing and half sized wing with applied distributed propulsion were obtained by CFD calculations in this study. Low-fidelity method was developed for fast preliminary design studies including studies of distributed propulsions. From this low-fidelity method the scale limit was derived.

Aerodynamic application of scaled wing can bring up to $38 \%$ save of fuel in cruise regime with predefined flight mission. The saved mass of fuel is mentioned to be used for 
DEP system and increasing of payload. This method doesn't account increase of mass for the wing due to installation of nacelles and also doesn't account increase of its drag. These constrains can be considered to be focused in future work. Increasing of drag can be solved for first approach by adding constant value of drag.

\section{Acknowledgement}

Authors acknowledge support from the EU Operational Programme Research, Development and Education, and from the Center of Advanced Aerospace Technology (CZ.02.1.01/0.0/0.0/16_019/0000826), Faculty of Mechanical Engineering, Czech Technical University in Prague.

\section{References}

1. Kim, H. D., Perry, A. T., and Ansell, P. J. (2018). A Review of Distributed Electric Propulsion Concepts for Air Vehicle Technology. 2018 AIAA/IEEE Electric Aircraft Technologies Symposium. doi:10.2514/6.2018-4998

2. Viken, J. K., Viken, S., Deere, K. A., and Carter, M. (2017). Design of the Cruise and Flap Airfoil for the X-57 Maxwell Distributed Electric Propulsion Aircraft. 35th AIAA Applied Aerodynamics Conference. doi:10.2514/6.2017-3922

3. Moore, K. R., and Ning, A., "Distributed Electric Propulsion Effects on Traditional Aircraft Through Multidisciplinary Optimization," AIAA Structures, Structural Dynamics, and Materials Conference, Kissimmee, FL, Jan. 2018. doi:10.2514/6.20181652

4. Gnadt, A., Isaacs, S., Price, R., Dethy, M., and Chappelle, C. (2019). Hybrid TurboElectric STOL Aircraft for Urban Air Mobility. AIAA Scitech 2019 Forum. doi:10.2514/6.2019-0531

5. Pavel, H., Jan, K., and Nikola, Ž. (2018). Wing and propeller aerodynamic interaction through nonlinear lifting line theory and blade element momentum theory. MATEC Web of Conferences, 233, 00027. doi:10.1051/matecconf/201823300027

6. EMRAX electrical engine, 2018, https://emrax.com/products/emrax-208/

7. Betz, A., Schraubenpropeller mit Geringstem Energieverlust, Göttinger Nachrichten, Gottingen, 1919, pp. 193-217.

8. Goldstein, S., On the Vortex Theory of Screw Propellers, Proc. of the Royal Society (A) 123, 440, 1929.

9. Larrabee, E. E., Practical Design of Minimum Induced Loss Propellers, Business Aircraft Meeting and Exposition, Society of Automotive Engineers, Warrendale, PA, April 1979, Paper 790595. 